Int. J. Dev. Biol. 58: 27-34 (2014)

doi: $10.1387 / \mathrm{ijdb} .130257 \mathrm{as}$

\title{
Zygotic expression of Exostosin1 (Ext1) is required for BMP signaling and establishment of dorsal-ventral pattern in Xenopus
}

\author{
YI-ER SHIEH ${ }^{1}$, DAN E. WELLS ${ }^{2}$ and AMY K. SATER ${ }^{*, 3}$ \\ Dept. of Biology and Biochemistry, University of Houston, Houston TX USA
}

\begin{abstract}
Exostosin 1 (EXT1) is a glycosyltransferase that contributes to the biosynthesis of heparan sulfate proteoglycans (HSPG). Loss of ext1 function leads to the human genetic disorder hereditary multiple exostoses (HME) and inhibits development in mouse, zebrafish and Drosophila. In Xenopus, loss of maternal EXT1 leads to impaired wnt11 signaling, resulting in a loss of dorsal embryonic development (Tao et al., 2005), but the functions of zygotic ext 1 have not been elucidated. In this study, morpholino oligonucleotides were used to generate a zygotic partial loss of function for ext1, in order to evaluate the requirements for ext1 function in gastrulation and paracrine signaling. Transcriptional profiling was carried out by microarray. Validation and subsequent analyses of gene expression were performed using Q-RT-PCR and in situ hybridization. Western blots were used to assess paracrine signaling pathway activity. Introduction of ext $1 \mathrm{MO}$ led to gastrulation defects, which were partially rescued by co-injection of ext1 mRNA. Microarray-based comparisons of gene expression in control vs. Ext1 MO embryos identified several developmentally significant genes that are dependent upon Ext1 function, including brachyury (Xbra). In addition, decreased Ext1 was shown to reduce the level of Wnt8 and BMP4 signaling and disrupt ventral-specific gene expression. Ext1 function is required for maintenance of normal levels of BMP and wnt, as well as their target genes. In addition, expression of $x b r a$ and the establishment of ventral mesoderm depend upon normal levels of Ext1. These findings suggest that ext1-dependent synthesis of HSPG is critical for wnt and BMP signaling, mesodermal identity, and ventral pattern.
\end{abstract}

KEY WORDS: exostosin, microarray, BMP, Xenopus, gastrula

\section{Introduction}

Exostosin1 and exostosin2 constitute a glycosyltransferase complex that is responsible for the polymerization of O-linked Heparan Sulfate (HS) chains (Ori et al., 2008). Since Heparan Sulfate Proteoglycans (HSPGs) have been implicated in several cell signaling pathways, including wnt, FGF, BMP, TGF $\beta$, and hedgehog, a reduction or loss of ext1 function is expected to have significant impact on a wide range of developmental processes. Mutations in human EXT1 are responsible for hereditary multiple exostosis (HME) (Ahn et al., 1995), now known as multiple osteochondromas, a genetic disorder characterized by multiple cartilaginous tumors.

Ext1 is critical for paracrine signaling via several pathways, as HSPGs are known to facilitate ligand-receptor interactions or act as low-affinity co-receptors (Ori et al., 2008). Mutations in the Drosophila orthologue of EXT1, tout-velu, disrupt Wingless signaling (Takei et al., 2004). Ext1 is indirectly implicated in BMP signaling, in that the HSPG Glypican 3 modulates BMP signaling in skeletal development and renal branching morphogenesis in mouse embryos (Paine-Saunders et al., 2000). HSPGs have also been extensively implicated in FGF signaling (reviewed in Matsuo and Kimura-Yoshida, 2013). Ext1 has specifically been identified

Abbreviations used in this paper: BMP, Bone Morphogenetic Protein; FGF, Fibroblast Growth Factor; GO, Gene Ontology; HME, Hereditary Multiple Exostoses; HSPG, Heparan Sulfate Proteoglycans; MAP Kinase, Mitogen-Activated Protein Kinase; MO, Morpholino Oligonucleotide; NCBI, National Center for Biotechnology Information; PCP, Planar Cell Polarity; PCR, Polymerase Chain Reaction; QRT-PCR, Quantitative Reverse Transcriptase Polymerase Chain Reaction; TCF, T-Cell Factor; TGF- $\beta$, Transforming Growth Factor - beta.

*Address correspondence to: Amy K. Sater. Dept. of Biology and Biochemistry, University of Houston, Houston TX USA 77204-5001.

Tel: 01-713-743-2688. Fax: 01-713-743-2636. E-mail: asater@uh.edu

Supplementary Material (table) for this paper is available at: http://dx.doi.org/10.1387/ijdb.130257as

Accepted: 7 November 2013. Final, author-corrected PDF published online: xx March 2014.

ISSN: Online 1696-3547, Print 0214-6282

(C) 2013 UBC Press

Printed in Spain 
as an indirect modulator of hedgehog signaling in both flies and vertebrates. Interestingly, mice heterozygous for ext2knockout show defects in cartilage and bone development that are independent of Indian Hedgehog (Ihh) signaling (Stickens et al., 2005).

Maternally expressed Ext 1 is required for wnt 11 signaling in early Xenopusembryos (Tao et al., 2005). Depletion of the maternal ext1 transcript results in reduced expression of the Wnt11 target genes goosecoid, siamois, chordin and Xnr3, leading to disruption of the dorsal-ventral axis (Tao et al., 2005). Mouse embryos display a similar early requirement for ext1; whole-embryo knockout of either ext1 (Lin et al., 2000) or ext2 (Stickens et al., 2005) is lethal, as homozygous knockout embryos fail to complete gastrulation.

This early maternal requirement for ext1 makes it difficult to assess the roles of ext1 during subsequent developmental processes. To evaluate functions of zygotically expressed ext1 during gastru- lation, we generated partial loss-of-function using a morpholino oligonucleotide $(\mathrm{MO})$ directed against ext 1 and evaluated the effects of ext1 knockdown on gene expression using microarray-based expression profiling. Our results indicate that ext1 is required for the completion of gastrulation and paracrine signaling via the wnt and BMP pathways. Interestingly, neither FGF nor nodal signaling is significantly affected by a reduction in ext 1 function.

\section{Results}

\section{Knockdown of Ext1 causes gastrulation defects}

In order to investigate the function of zygotic ext1 in early gastrulation, we developed a morpholino oligonucleotide directed against ext1 (EXT1 MO), as well as a 5-base mispair MO (EXT1 MIS). To evaluate the efficacy of the morpholino oligonucleotides,
A

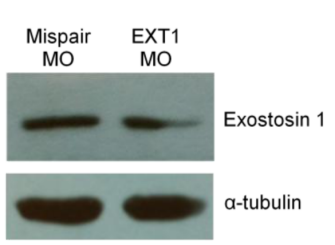

B

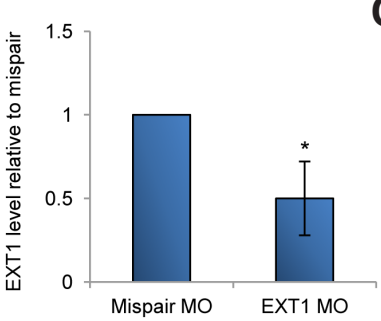

C

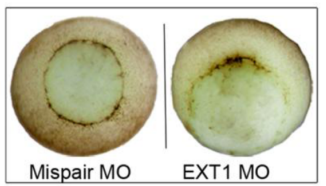

E
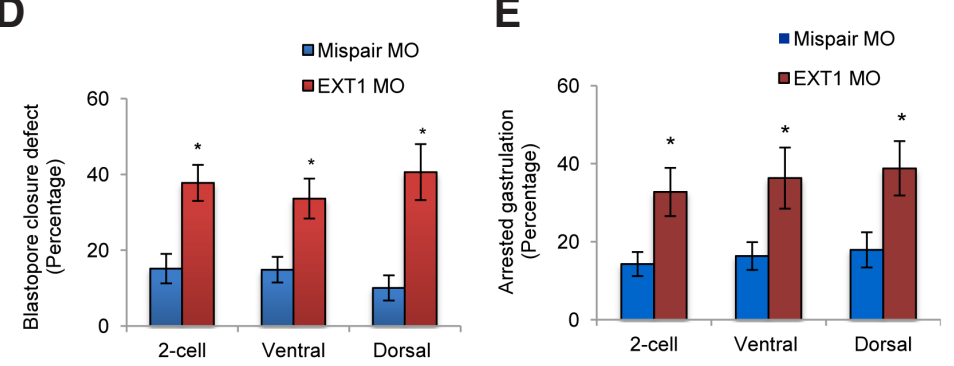

$\mathbf{F}$

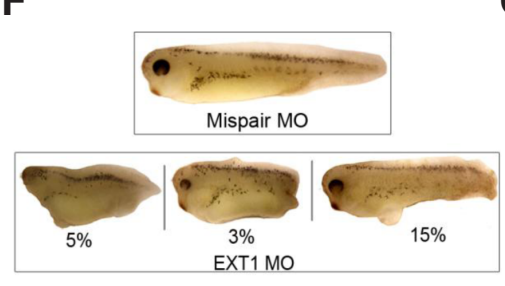

I

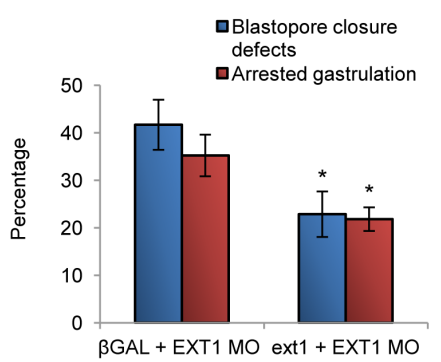

G

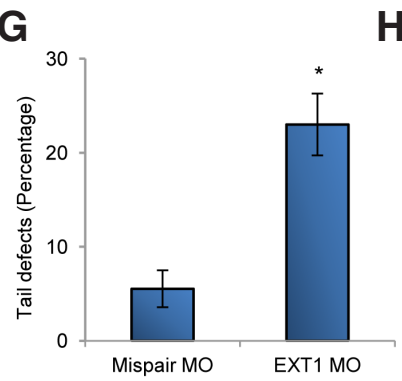

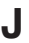

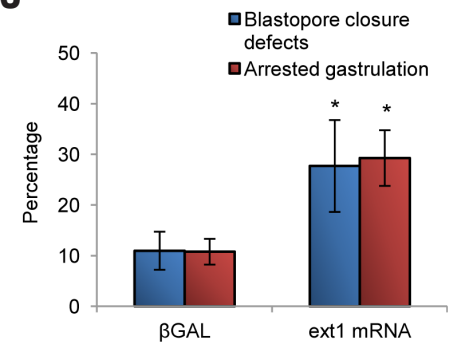

Fig. 1. Knockdown of ext1 results in gastrulation defects. (A) $E m$ bryos were injected with $20 \mathrm{ng}$ of either the EXT1 MO or the mispair MO and lysed at st. 10.5 in preparation for immunoblotting to detect Ext1 and $\alpha$-tubulin (loading control). Ext1 protein levels are decreased in EXT1-MO injected embryos. (B) Quantitative comparison of Ext1 protein accumulation. Relative Ext1 levels were normalized with $\alpha$-tubulin. Bars represent mean \pm S.D. $N=3$ independent experiments. (C) Midgastrula EXT1 MO-injected embryos exhibited incomplete blastopore formation and partialendoderm protrusion, whereas the mispair MO-injected embryos had a normal blastopore. (D,E) Embryos were injected with 20 ng of mispair or EXT1 MO at either the 2-cell stage or in either dorsal or ventral cells at the 4- cell stage; the phenotypes were compared at midgastrula (St. 10.5). A higher percentage of blastopore closure defects (D) or gastrulation arrest (E) were observed in EXT1 MO-injected embryos, irrespective of the stage or location of injection. $N=$ 10 experiments, with a total of 400 embryos for each condition. (F) The phenotypes of embryos with head and tail defects in ext1 morphants. (G) The frequency of tail defects in ext1 morphants. Total number of tadpoles was 213. Bars present meant \pm S.D. $\mathrm{N}=7$ independent experiments. (H) The survival rates for EXT1 MO and EXT1 MIS embryos. Bars represent mean $\pm S$.D. $N=7$ independentexperiments (20-60 embryos/injection). (I) Blastopore closure defects and arrested gastrulation in ext1 morphants were partially rescued by coinjection with ext 1 mRNA. Bars present meant

$\pm S$.D. $N=5$ independent experiments. (J) Embryos injected with 1 ng of $\beta$-galactosidase or ext1 mRNA were collected and phenotypes were compared at gastrulation stage. The embryos overexpressing ext1 had higher frequencies of blastopore defects and gastrulation arrest compared to controls. Bars present meant \pm S.D. $N=5$ independent experiments. ${ }^{*} p<0.05$. 
A

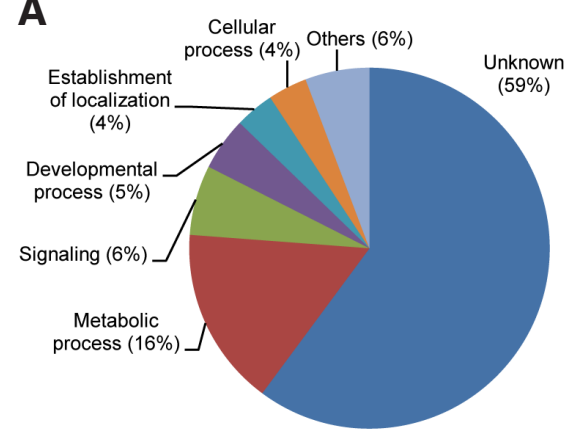

B

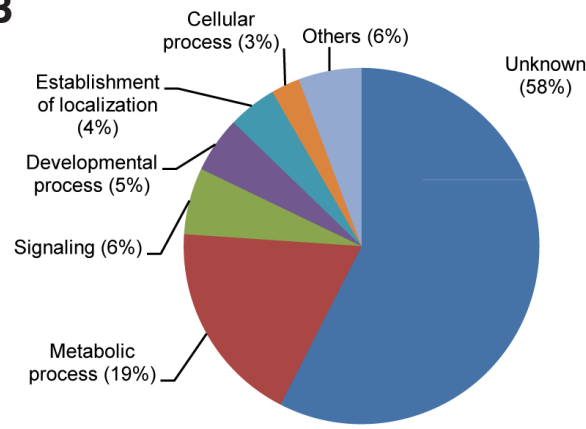

Fig. 2. Gene ontology (GO) of genes showing dysregulation in ext 1 morphants. We characterized genes that showed a change of greater than two fold (A) or less than 0.5 fold (B) in ext1 morphant embryos. More than half of the genes up-regulated in response to ext1 knockdown had unknown functions. Among those with known functions, 16\% were associated with metabolic processes, including transcriptional and translational regulation. Signaling-associated genes accounted for $6 \%$, while $5 \%$ were involved in

developmental processes. The remainder included establishment of localization (4\%), cellular processes (3-4\%), and others (6\%). The "others" category includes multicellular organismal processes, response to stimulus, cell proliferation, cellular component organization, and reproductive processes. Similar proportions were observed for genes that were down-regulated following a reduction in Ext1 expression.

$20 \mathrm{ng}$ of each $\mathrm{MO}$ were injected into embryos in preparation for immunoblotting to evaluate the accumulation of Ext1 protein in injected gastrula embryos. The results showed that in EXT1 MOinjected embryos, Ext1 protein levels are reduced to approximately $50 \%$ when compared to embryos injected with EXT1 MIS (Fig. 1 $A, B)$. This concentration was also shown to produce phenotypic effects with EXT1 MO, but not EXT1 MIS; it was used in subsequent experiments, as injection of higher concentrations led to nonspecific effects.

The Ext1 knockdown embryos showed abnormal gastrulation compared to uninjected or EXT1 MIS-injected embryos. Specifically, the blastopore of Ext1-deficient embryos had incomplete, irregular closure and extrusion of endoderm (Fig. 1 C,D). In addition to an incomplete or abnormal blastopore, Ext1 knockdown embryos also exhibited arrested gastrulation (Fig 1E). Targeted injections of EXT1 MO into either dorsal or ventral blastomeres at the 4 cell stage also resulted in incomplete blastopore closure, (Fig. 1D) and a significant increase in gastrulation arrest (Fig. 1E) compared with embryos injected with the mispair morpholino. These phenotypes suggest that Ext 1 is required for normal gastrulation. Moreover, this requirement is not restricted to dorsal- or ventral-specific tissues.

Although there are no significant phenotypic abnormalities that emerge immediately following gastrulation, additional defects in the ext1 morphants appear after neurulation. Embryos injected with EXT1 MIS had long straight tails, while ext1 morphant embryos had a range of tail defects in nearly $25 \%$ of cases (Fig. 1 F-G). In approximately $1 / 3$ of these embryos, anterior structures were reduced or absent (Fig. 1F). Knockdown of ext1 also leads to increased mortality at later stages. The survival rate of ext1 morphants drops significantly when the embryos start to elongate following neural tube closure (Fig. 1H).

We coinjected the EXT1 MO with mRNA encoding either ext1 or $\beta$-galactosidase to determine whether overexpression of ext 1 would rescue the morphant phenotype. Ext1 overexpression lowered the frequency of blastopore closure defects by approximately half and also reduced the incidence of gastrulation arrest as well (Fig. 1l). This result confirms the specificity of the EXT1 MO.

We evaluated the effects of ext1 overexpression by injecting 2 -cell embryos with $1 \mathrm{ng}$ of mRNA encoding either $\beta$-galactosidase (control) or ext1; embryos were observed throughout gastrulation. Overexpression of ext 1 led to incomplete blastopore closure and arrested gastrulation (Fig. 1J), similar to the phenotypes observed in ext1 knockdown embryos. The similarity between the overexpres- sion and knockdown phenotypes suggests that the optimal level of ext1 expression may reflect a ratio of Ext1 to other proteins, rather than an absolute or independent level. This similarity may also explain why coinjection of the EXT1 MO and ext1 mRNA leads to a partial rescue.

\section{Identification of genes responsive to changes in Ext1 expression}

Since ext1 has been implicated as an indirect contributor to multiple signaling pathways in vertebrate development, we sought an unbiased approach to identify genes expressed in gastrulae that would be affected by a reduction in ext1 expression. Therefore, we carried out a microarray analysis to identify such genes. Embryos were injected with $20 \mathrm{ng}$ of either EXT1 MO or EXT1 MIS at the two-cell stage, and mRNA was isolated when the injected embryos reached early gastrula (stage 10.25). The RNA samples were compared across 3 biological replicates using the Affymetrix Xenopus laevis genome 2.0 Gene Chip.

Ext1-regulated genes were defined as genes represented by probe sets that showed $a \geq$ two-fold change in expression across three independent biological replicates. We identified 405 transcripts up-regulated more than 2-fold and 609 transcripts down-regulated less than 0.5 -fold out of approximately $\sim 15,000$ transcripts (See Supplementary Table 1); these transcripts were evaluated by gene ontology. The NCBI Unigene cluster assigned to the Affymetrix Xenopus laevis probe set was linked to the human orthologue. The human orthologues of each gene were then used as the basis for the gene ontology analysis (http://www.geneontology.org). Data collected from Gene Ontology analysis revealed that more than half of the genes affected by Ext1 knockdown were of unknown function. Among the remaining genes, the following categories were strongly represented: metabolic processes (including transcription and translation), signaling, developmental process, establishment of localization, cellular process, multicellular organismal process, response to stimulus, cell proliferation, cellular component organization, and reproductive process (Fig. 2). Most of the affected genes with known functions have primary activities unrelated to developmental processes.

Genes with known roles in embryonic development were selected for subsequent validation by q-RT-PCR. Five up-regulated genes (is/1, pitx2, tbx5A, wnt5A, wnt7A) and four down-regulated genes (Xbra, pax3, wnt1, WT1) were assayed (Fig. 3A). Significant differences in expression were found for Xbra, is/1, pitx2, wnt1, and 
wnt7A, but not for pax3, tbx5A, wnt5A, and WT1.

\section{Ext1 and paracrine signaling pathways}

Given the abnormal gastrulation phenotype, we were particularly interested in the finding that the knockdown of Ext1 leads to a down-regulation of Brachyury (xbra) expression, since xbraactivity is required for mesoderm formation, notochord development, and normal gastrulation in mouse, zebrafish and Xenopus (reviewed in Smith, 2004). During gastrulation, $x$ bra is expressed throughout the marginal zone and is present in the notochord and tail tissue during neurula and tailbud stage (reviewed in Smith, 2004). We confirmed the downregulation of xbra expression in gastrula embryos via in situ hybridization (Fig. 3B).

$X$ bra activates transcription of $e F G F$, which maintains expression of $x b r a$, thus establishing a positive feedback loop; several other signaling pathways, including activin/nodal, BMP, and canonical wnt, are also known to activate and/or maintain expression of $x b r a$ (reviewed in Smith, 2004). Weassessed expression of eFGF, as well as another direct $x$ bra target, wnt11, via Q-RT-PCR; eFGF expression is dramatically reduced in ext1 morphant embryos, relative to
A

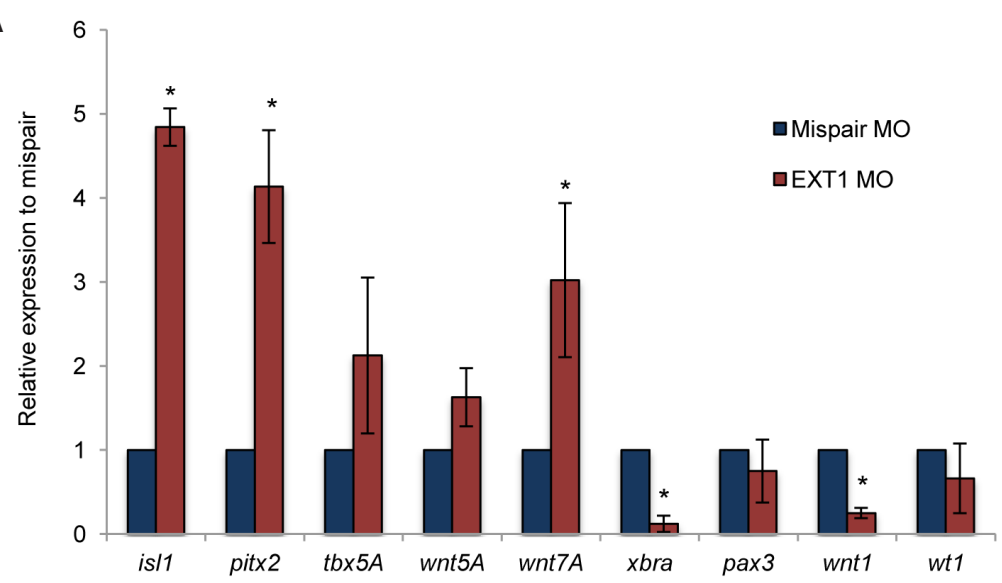

B

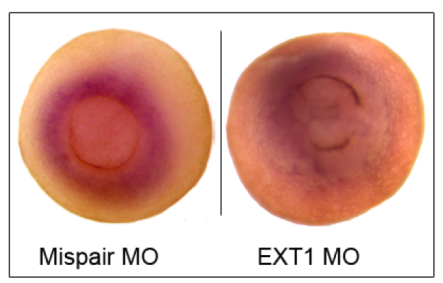

C

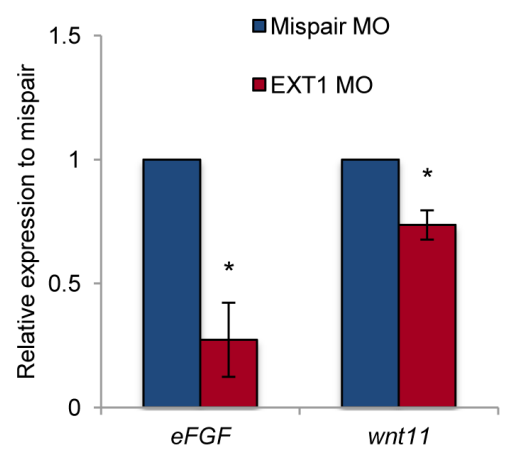

Fig. 3. Validation of $x$ bra and other genes affected by ext1 knockdown. (A) Selected genes identified by microarray were validated using Q-RT-PCR. Embryos injected with $20 \mathrm{ng}$ of EXT1 MO or EXT1 MIS were collected at stage 10.5 for RNA extraction and Q-RT-PCR. (B) In situ hybridization shows differences in xbra expression in control vs ext1 morphant embryos. Results are representative of over $2 / 3$ of embryos from 3 independent experiments. (C) Q-RT-PCR assays for expression of the xbra target genes eFGF and Wnt11. Relative fold enrichment of each target gene was calculated after normalization with the housekeeping gene ornithine decarboxylase (ODC) using the $\triangle \Delta C$ t method. Bars represent mean $\pm S . D . N=3$ independent experiments. ${ }^{*}: p<0.05$. controls (Fig. 3C). Expression of wnt11 is also decreased, albeit to a lesser degree. This reduction in wnt11 expression may account for the gastrulation defects, since wnt11 is required for appropriate coordination of mesodermal cell movements during gastrulation.

Since several paracrine pathways contribute to the regulation xbra expression, we initially asked whether these pathways are Signal Regulated (ERK) Mitogen Activated Protein (MAP) kinase was altered in ext1 morphant embryos (Fig. 4 A,B). Quantification 列 that there is no discernible difference in ERK MAP kinase phosphorylation. A similar lack of effect was observed with immunoblots probed with antibodies to Smad3 and phospho-Smad3, which would neither FGF/MAPK nor nodal signals are impaired across the embryo by a reduction in Ext1 function.

We also evaluated the effects of Ext1 knockdown on BMP signaling by comparing levels of activated (phospho-) Smad1 in control and ext1 morphant embryos. MO-mediated reduction in Ext1 decreased the amount of phospho-Smad1 by approximately $50 \%$ (Fig. 4C). We also assessed the expression of the BMP4 target genes vent1, vent2, and msx1 in ext1 morphant embryos via Q-RT-PCR; BMP4 itself was also included, since BMP4/Smad1 signals activate $B M P 4$ transcription, forming an autoregulatory loop. In ext1 morphant embryos vent1, vent2, msx1 and BMP4 are down-regulated (Fig. 4D), and BMP4 shows a dramatic reduction to less than $10 \%$ of levels observed in controls. These findings demonstrate that ext1 is required for BMP4 signaling and the maintenance of BMP4 expression.

Overexpression studies suggest that BMP4 is sufficient (Northrup et al., 1995) but not necessary (Kumano and Smith, 2000) for xbra expression. Thus, these findings do not account for the reduction of $x b r a$ in ext1 morphant embryos. Wnt8, however, is an upstream regulator of $x$ bra at late blastula and gastrula stages (Vonica and Gumbiner, 2002): binding sites for TCF have been found in the xbra promoter, and mutation of TCF3 decreases the expression of $X b r a$, suggesting that $x b r a$ is a direct target of canonical Wnt signaling (Vonica and Gumbiner, 2002). We assessed expression of wnt8and the wnt8 target genes myoD, HoxA1, HoxB1, and HoxD1 in ext1 morphant embryos; expression of these genes is substantially reduced in the morphants, and expression of wnt8 decreases to approximately $10 \%$ of controls (Fig. 4E).

\section{Ext1 and dorsal-ventral pattern}

To understand how a reduction in ext1 affects dorsal-ventral patterning during gastrulation, we examined the expression of the organizer genes not 1 and otx2 and the ventrally expressed BMP target genes vent 1 and vent2. As expected from our earlier Q-RT-PCR results, the regions expressing vent1 and 
vent2 are diminished in the ext1 morphants, and the overall level of expression is also reduced (Fig. 5 A-D). Interestingly, however, there is no corresponding expansion of expression for the organizer genes; indeed, expression of the organizer genes not 1 and otx2 is diminished across the dorsal region (Fig. 5 E-H). Organizergene expression was also evaluated via Q-RT-PCR; expression levels for the organizer genes chordin, goosecoid, and ot $x 2$ are decreased by over $50 \%$ in ext1 morphants.

\section{Discussion}

Our results indicate that a reduction in the expression of Ext1 leads to defects in gastrulation. This conclusion is consistent with earlier findings indicating that HSPGs are required for both the cell migratory behavior characteristic of the head mesoderm (Smith et al., 2009) and for the convergence extension movements observed in the axial mesoderm (Ohkawara et al., 2003). Gain or loss of the Xenopus HSPGs syndecan-4 (xSyn4) or glypican4 (xgly4) result in convergent extension defects in activin-treated animal caps and a shortened anteroposterior axis in intact embryos (Ohkawara et al., 2003; Munoz et al., 2006). The zebrafish mutation knypek represents a loss of function of glypican4; mutant embryos show impaired convergent extension movement and abnormal cell polarity (Sepich et al., 2011). Moreover, since HSPGs are essential for wnt signaling, loss of ext 1 function might disrupt the non-canonical wnt signals that regulate the planar cell polarity (PCP) pathway responsible for the coordination of cell behavior during convergent extension. It should be noted that although expression of wnt11 is decreased, the major components of the PCP pathway do not show changes in expression in ext1 morphant embryos (Suppl. Table 1). Impairment of HSPG synthesis and the resulting reduction in wnt11, however, presumably lead to attenuated signaling through the PCP pathway, which could underly the observed defects in gastrulation.

The T-box gene brachyury ( $x b r a$ ) is required for mesoderm formation and the completion of gastrulation (reviewed in Smith, 2004). Our transcriptional profiling revealed that $x b r a$ is down-regulated in ext1-morphant embryos. Similarly, in EXT1 knockout mouse embryos, mesoderm does not form and brachyuryexpression is not detected (Lin etal., 2000).

Xbraexpression is initiated in vivo by nodal-related signals, and maintained in large part via a positive feedback loop involving transcriptional activation of eFGF. Our results indicate that neither the nodal/ activin pathway nor the FGF/erk MAPK pathway show reduced activity in ext1 morphant embryos, which suggests that $X b r a$ expression is decreased in spite of nodal-related or FGF signaling. Wnt signals also contribute to the activation and maintenance of Xbra expression (Vonica and Gumbiner, 2002), so

\section{A}

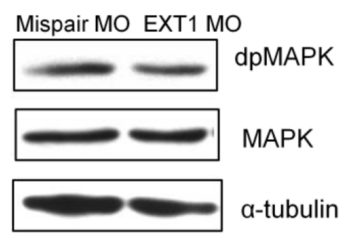

B

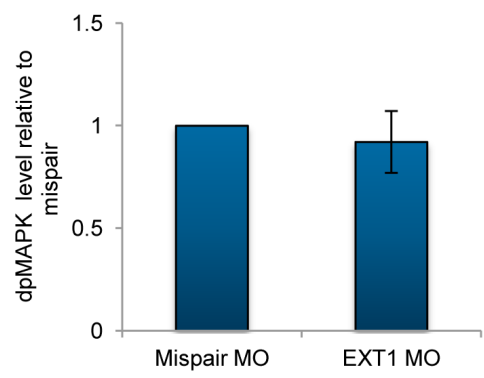

C

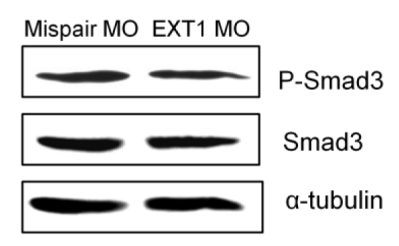

D

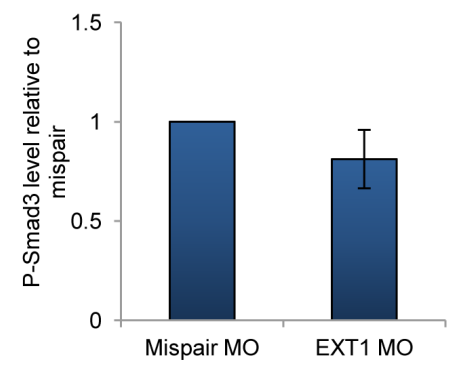

$\mathbf{F}$

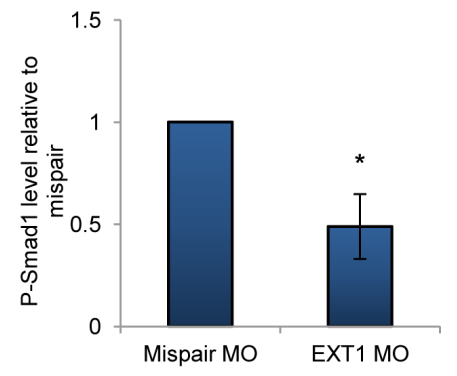

H
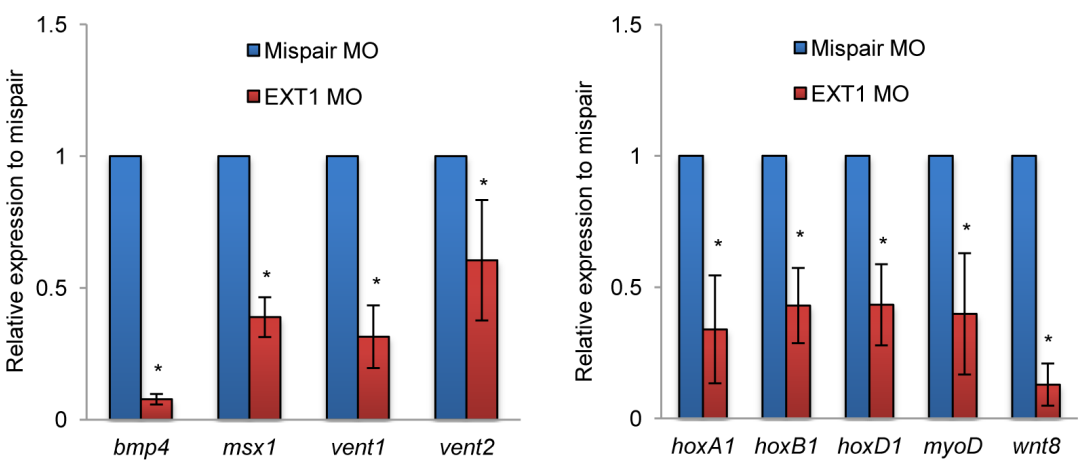

Fig. 4. The effects of Ext1 knockdown on paracrine signaling pathways. Embryos were injected with EXT1 or mispair MO and lysed at stage 10.5. (A,C,E) Immunoblots showed levels of diphospho- Erk MAPK (dpMAPK), phospho-Smad3, and phospho-Smad1 in EXT1 or mispair MO-injected embryos. (B,D,F) Relative levels of phosphorylated forms of MAPK, Smad3, and Smad1 in EXT1 or mispair MO-injected embryos. Phospho-Smad1 is reduced in EXT1 morphant embryos, whereas levels of dpMAPK and phopho-Smad3 are unchanged. (G) Q-RT-PCR assays of BMP4 and the BMP4 target genes msx1, vent1, and vent 2 in control or ext1 morphant embryos. (H) Q-RT-PCR assays of wnt8 and the wnt8 target genes HoxA1, HoxB1, HoxD1, and myoD. The relative -fold change of each target gene in EXT1 MO-injected embryos was calculated as described in Fig. 3. $(B, D, F-H)$ Bars represent mean $\pm S . D . N=3$ independent experiments. ${ }^{*}: p<0.05$. 

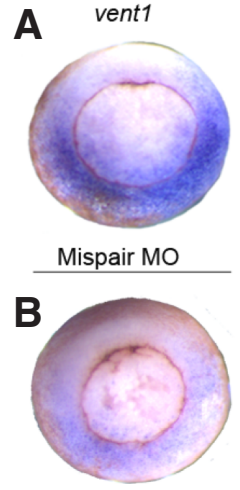

EXT MO
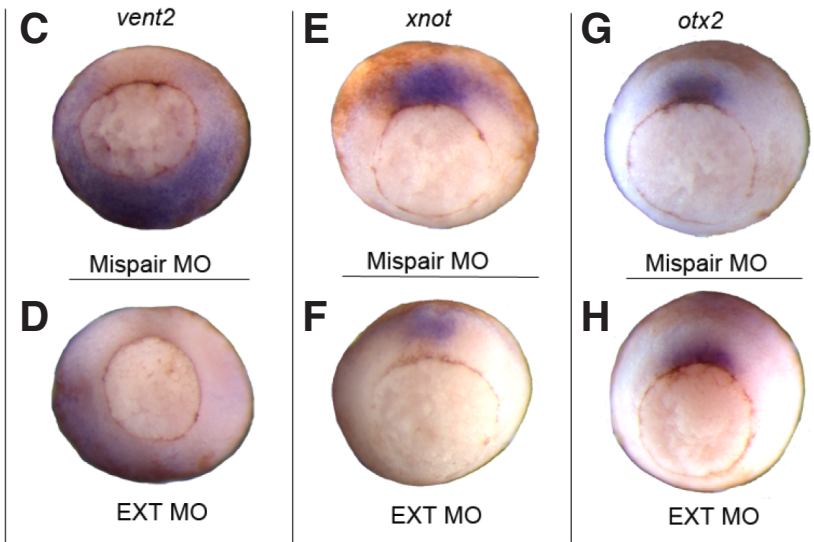

|

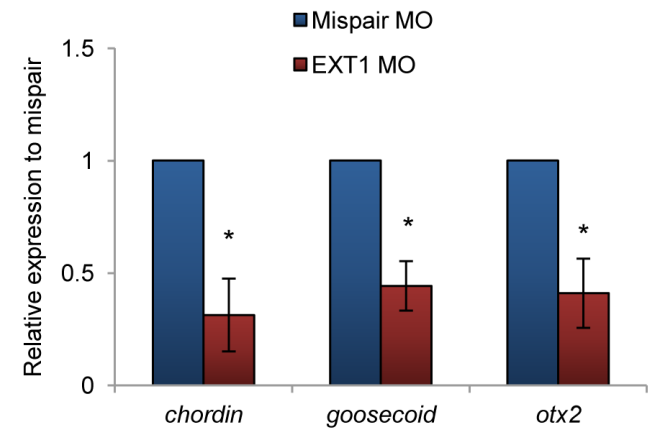

Fig. 5. Establishment of dorsal- or ventral-specific patterns of gene expression in ext1 morphant embryos. Embryos were injected with $20 \mathrm{ng}$ of EXT1 or mispair MO. In situ hybridization of vent1 (A,B); vent2 (C,D); not1 (E,F); and otx2 (G,H). Expression patterns shown are representative of at least 2/3 of embryos from 3 independent experiments. (I) Q-RT-PCR assays of organizer-specific gene expression in ext1 $\mathrm{MO}$ or mispair MO embryos. Relative-fold changes were evaluated as described in Fig. 3. Bars represent mean \pm S.D. $N=3$ independent experiments. ${ }^{*}: p<0.05$.

decreased wnt signaling activity may account for the reduction in Xbra expression in ext1 morphants.

A related conundrum offered by these findings is that erk MAPK phosphorylation is unaffected, despite a dramatic reduction in the expression of eFGF. However, erk MAPK activity in gastrula embryos reflects inputs from other FGFs, as well as stimulation by Insulin-like Growth Factor (IGF) and other receptor tyrosine kinasedependent paracrine signals. Thus, while eFGF activity may be depressed in the marginal zone, this may have only a limited effect on the erk MAPK activation averaged across the entire embryo.

Brachyury has been shown to play a critical role in the regulation of specific cell behaviors during vertebrate gastrulation (reviewed in Smith, 2004): in mouse embryos, cells lacking functional brachyury were unable to migrate out of the primitive streak, while in Xenopus, $x b r a$ function is required for the mediolateral intercalation behavior that underlies convergentextension (Conlon and Smith, 1999; Kwan and Kirschner, 2003). Brachyury function is not required for the migratory behavior characteristic of anterior mesoderm, however (Conlon and Smith, 1999; Kwan and Kirschner, 2003). Thus, the gastrulation defects observed in ext1 morphantembryos may result directly from the reduction in Xbra expression.

The transcriptome comparisons of Yanai et al., (2011) indicate that expression of $X$. laevis ext 1 mRNA drops by nearly $20 \%$ between st. 9 and the end of gastrulation; this decrease presumably reflects the loss of the maternal ext1 transcript. Our results indicate that an additional loss of $50 \%$ of the total accumulated Ext1 protein is sufficient to disrupt signaling through at least two major paracrine signaling pathways, wnts and BMPs. The timing of the drop in ext 1 transcript accumulation is noteworthy because heparan sulfate accumulation increases significantly during gastrulation (Yamada et al., 2009), and HSPGs are required for both signaling and cell movement during gastrulation. Thus, gastrulation may be a period of increased sensitivity to reductions in ext1 accumulation or activity. Although depletion of maternal ext1 does not affect expression of vent1 (Tao et al., 2005), our results indicate that a loss of zygotic expression leads to decreased vent1 expression. This difference suggests that the critical interval, during which HSPGs are required for the BMP signals that activate vent1, follows the loss of maternal transcripts after the onset of gastrulation.

Our findings suggest that ext1 function is required for the cellular signals that establish dorsal-ventral pattern within the mesoderm. Although primary pathways for both FGF and nodal-related signals are apparently unaffected, reduction in Ext1 expression disrupts both BMP and wnt signals. Biochemical studies have demonstrated that BMPs bind to HSPG (Ohkawara et al., 2002), and knockdown of HSPG results in reduction of BMP signaling in Xenopus early development (Olivares et al., 2009). The HSPG glypican3 modulates BMP signaling (Paine-Saunders et al., 2000), and Wnt8 and Wnt11 bind directly to glypican4 (Ohkawara et al., 2003). Tissue-specific knockout of EXT1 leads to abnormalities in BMP signaling in mouse skeletal development (Matsumoto et al., 2010).

In ext1 morphants, both dorsal and ventral tissue identities are affected, as shown by a reduction in tissue-specific gene expression. The effects on ventral identity are more pronounced, as shown by the loss of regionalized expression of vent2: both BMP4 and Wnt8 regulate patterning of ventral mesoderm (Hoppler and Moon, 1998) and both pathways are impaired in ext1 morphant embryos. In contrast, organizer-specific gene expression is still properly regionalized, reflecting proper function of the maternal wnt11 pathway, although expression levels are lower than in controls. This may result from the reduction in Xbra and the concommitant loss of mesoderm.

Our results add to the already extensive findings demonstrating roles for HSPGs and the enzymes responsible for their biosynthesis and modulation during early vertebrate development. Syndecans and glypicans have been strongly implicated as low-affinity receptors for several paracrine signals (Matsuo and Kimura-Yoshida, 2013), as regulators of the distribution of paracrine signals (Billoni et al., 2013), and as modulators for the shape of morphogen gradients in embryonic tissues (Yan and Lin, 2009). Perlecan promotes shh localization and activity (Palma et al., 2011). Moreover, the extracellular HSPG remodeling endosulfatases contribute to the regulation of wnt, BMP, and FGF signals (Freeman et al., 2008). Additional studies have demonstrated that heparan sulfate itself regulates pluripotency and self-renewal in embryonic stem cells (e.g., Sasaki et al., 2008). HSPGs and their modulators are essential 
components for the fine-tuning of cell signaling and cell behavior.

\section{Materials and Methods}

\section{Embryos collection and treatments}

Embryos were obtained by the methods described in Sive et al., (2000). Morpholino oligonucleotides were obtained from Gene Tools. Embryos were injected at the 2- or 4-cell stage with $20 \mathrm{ng} / \mathrm{embryo}$ of either the ext1 MO (5' - AGCGTTTTTTCGCCTGCATGTGTCC - 3') or the mispair MO (5'AGGGTATTTTCCCCTGGATGTCTC -3' - altered bases are underlined). Capped mRNAs for microinjection were synthesized using the mMessage mMachine in vitro transcription kit (Applied Blosystems).

\section{Microarray}

For each sample, RNA was isolated from five embryos at st 10.5 using the RNeasy mini kit (Qiagen). RNA samples were prepared from three independent sets of ext1 MO- and mispair MO- injected embryos. Probe synthesis and hybridization to Affymetrix Xenopus laevis genome 2.0 GeneChip were performed by the Baylor College of Medicine Microarray Core Facility. The mispair and ext1 MO-injected samples were processed in parallel to reduce inter-sample variation. Raw CEL and DAT files were generated using Affymetrix Microarray Suite 5.0 (MAS 5). The values for the control and experimental array were normalized, and the -fold change of each gene between mispair and ext1 MO was calculated. Genes for which the standard deviation exceeded the -fold change were omitted from further consideration. Those genes with a -fold change bigger than 2 or smaller than $\mathbf{0 . 5}$, were chosen for further analysis. Gene Ontology (GO) analysis of each gene was carried out by using the NCBI Unigene set to identify the human orthologue, which was used as the basis for GO analysis (GO Consortium; http://www.geneontology.org).

\section{Quantitative RT-PCR (Q-RT-PCR)}

Embryos were lysed in TRIzol (Invitrogen), and RNA was isolated according to manufacturer's instructions. For each sample, 2 micrograms of total RNA were treated with DNase (RQ1 RNase-free DNase, Promega); cDNA synthesis was performed using either the superscript III (Invitrogen) or First-Strand cDNA Synthesis Kit (Roche). The qPCR primer sequences are listed below:

BMP-4 (F): GCATGTAAGGATAAGTCGATC; BMP-4 (R):GATCTCAGACTCAACGGCAC HoxA1 (F): ACCAGCAGCACCAGTCTTAC; $\operatorname{HoxA1}(\mathrm{R})$ : GGAGGCTGGATACATTGTTG $\operatorname{HoxB1}$ (F): GATGGAAGGTTTGTTGTTGG; $\operatorname{HoxB1}$ (R): GTGAGGGTAACTGGGGTTCT HoxD1 (F): TTTCCAGGCAATGGATCTTA; HoxD1 (R): GCCCCCAGGATAAACTTCTA is/1 (F): GGGTCAGGACGGTTCTTAAT; is/1 (R): ACGAGCTGTTCCTTCATCAG, myoD (F): TTGAGACCCTGAAGCGATAC; myoD (R): TAATGTTCCAGAACCGGGTA $m s \times 1(\mathrm{~F})$ : ACTGGTGTGAAGCCGTCCCT; $m s x 1$ (R): TTCTCTCGGGACTCTCAGGC pax3 (F): CGCTAGATGGAGAAAGCAAG; pax3 (R): CTGGCATAGCTGTAGGAGGA pitx2 (F): CAGTGTGGACCAATCTGACA; pitx2 (R): TGGTTCCTCTCCCTCTTTCT tbx5A (F): CATCCTGAATTCAATGCACA; tbx5A (R): CAAAGCCATTGTTCTCGTCT vent1 (F): TGGTTCAACAGGGATTCTC; vent1 $(\mathrm{R})$ : CTGCTAAGGAAGGATTTGC vent2 (F): CCTCTGTTGAATGGCTTGCT; vent2 (R): TGAGACTTGGGCACTGTCTG wnt1 (F): AATGGTGGGGGATAGTGAAT; wnt1 (R): TCTAGCACCAAAGGAACAGG wnt5A (F): TTAAAACCTGTTGGCTCCAG;
wnt5A (R): CGCACTGTCGTATTTCTCCT

$w n t 7 A(\mathrm{~F})$ : GAGAGCAAGCAGAAACAAGC; $w n t 7 A(\mathrm{R})$ : TAGACCAGATCGGTGTCCAT WT1 (F): CCTTGACCCCTCATTCTTTT; WT1 (R): TAAATGCGCTGAGACACTGA xbra (F): AGACATCTTGGATGAGGG; xbra (R): GAAGGGTACTGACTTGAG

\section{Whole mount in situ hybridization}

In situ hybridization was carried out according to Sive et al., (2000). The antisense RNA probes were synthesized and labeled with digoxygenin using the Maxiscript kit (Applied Biosystems).

\section{Western Blot}

For each sample, 10 embryos were lysed at st. 10.5 in $100 \mu \mathrm{l}$ Kinase Buffer (20 mM HEPES pH7.5, 40 mM MgCl, 20 mM EGTA, 1 mM DTT, 80 mM glycerol-2-phosphate, $50 \mathrm{mM} \mathrm{NaF}, 1 \mathrm{mM}$ sodium orthovanadate, $1 \mathrm{mM}$ microcystin, and 2.5X protease inhibitor cocktail). Samples were centrifuged at $13,000 \mathrm{rpm}$ for 20 minutes in $4^{\circ} \mathrm{C}$ and then prepared for SDS-PAGE. Following electrophoresis, the protein was transferred from gel to membrane using a Bio-Rad semi-dry apparatus. The membrane was incubated with primary antibody overnight in $4^{\circ} \mathrm{C}$. The primary antibodies are Exostosin 1 (Abbiotech, 1:100); Phospho-Smad1 (Cell Signaling, 1:500); Smad1 (Abcam, 1:1000); Phospho-Smad3 (Cell Signaling, 1:1000); Smad3 (Cell Signaling, 1:1000), and $\alpha$-tubulin (Abcam, 1:2000). Proteins were visualized using HRP-conjugated secondary antibodies (Sigma-Aldrich) and the ECL plus kit (Amersham/ GE Healthcare Life sciences). The intensity of each band on the membrane was quantified using the "area measurement" function of Image J (Schneider et al., 2012), a Java-based image processing program (http://rsb.info.nih.gov/ij/index.html).

\section{Accession number}

The microarray data have been deposited into the NCBI Gene Expression Omnibus and are available under accession number GSE51562.

\section{Acknowledgements}

We would like to thank B. Bodmann, A. Benham, and J. Songhurst for advice and assistance with the statistical analysis of microarray data, $K$. Cho, J. C. Smith, C. Niehrs, P. McCrea, and D. Kimelman for plasmids; C.-H. Lou, C. Liu, R. Ritter for comments and suggestions, and J. Talley for technical assistance. Microarrays were performed by the Microarray Core Facility, Baylor College of Medicine. This work was supported by an award from the Multiple Hereditary Exostoses Research Foundation to D.E.W. and NSF award IOS-1147047 to A.K.S.

\section{References}

AHN J, LUDECKE H J, LINDOW S, HORTON W A, LEE B, WAGNER M J, HORSTHEMKE B, WELLS D E. (1995). Cloning of the putative tumour suppressor gene for hereditary multiple exostoses (EXT1). Nat Genet 11: 137-143.

BILIONI A, SÁNCHEZ-HERNÁNDEZ D, CALLEJO A, GRADILLA AC, IBÁÑEZ C MOLLICAE, CARMEN RODRÍGUEZ-NAVASM, SIMONE, GUERREROI. (2013). Balancing Hedgehog, a retention and release equilibrium given by Dally, Ihog, Boi and shifted/DmWif. Dev Biol 376: 198-212.

CONLON F L, SMITH J C. (1999). Interference with brachyury function inhibits convergent extension, causes apoptosis, and reveals separate requirements in the FGF and activin signalling pathways. Dev Biol 213: 85-100.

FREEMAN SD, MOORE WM, GUIRALE C, HOLMEAD, TURNBULLJ E, POWNALL $M E$. (2008). Extracellular regulation of developmental cell signaling by XtSulf1. Dev Biol 320: 436-445

HOPPLER S, MOON R T. (1998). BMP-2/-4 and Wnt-8 cooperatively pattern the Xenopus mesoderm. Mech Dev 71: 119-129.

KUMANO G, SMITH W C. (2000). FGF signaling restricts the primary blood islands to ventral mesoderm. Dev Biol 228: 304-314. 


\section{Y.-E. Shieh et al.}

KWAN K M, KIRSCHNER M W. (2003). Xbra functions as a switch between cell migration and convergent extension in the Xenopus gastrula. Development 130: 1961-1972.

LIN X, WEI G, SHI Z, DRYER L, ESKO J D, WELLS D E, MATZUK M M. (2000). Disruption of gastrulation and heparan sulfate biosynthesis in EXT1-deficient mice. Dev Biol 224: 299-311.

MATSUMOTOY, MATSUMOTOK, IRIE F, FUKUSHIJ, STALLCUPWB, YAMAGUCHI Y. (2010). Conditional ablation of the heparan sulfate-synthesizing enzyme Ext1 leads to dysregulation of bone morphogenic protein signaling and severe skeletal defects. J Biol Chem 285: 19227-19234.

MATSUO I, KIMURA-YOSHIDA C. (2013). Extracellular modulation of Fibroblast Growth Factor signaling through heparan sulfate proteoglycans in mammalian development. Curr Opin Genet Dev 23: 399-407.

MUNOZ R, MORENO M, OLIVA C, ORBENES C, LARRAIN J. (2006). Syndecan-4 regulates non-canonical Wnt signalling and is essential for convergent and extension movements in Xenopus embryos. Nat Cell Biol 8: 492-500.

NORTHROPJ, WOODSA, SEGERR, SUZUKIA, UENON, KREBSE, KIMELMAND. (1995). BMP-4 regulates the dorsal-ventral differences in FGF/MAPKK-mediated mesoderm induction in Xenopus. Dev Biol 172: 242-252.

OHKAWARA B, IEMURA S, TEN DIJKE P, UENO N. (2002). Action range of BMP is defined by its N-terminal basic amino acid core. Curr Biol 12: 205-209.

OHKAWARA B, YAMAMOTO T S, TADA M, UENO N. (2003). Role of glypican 4 in the regulation of convergent extension movements during gastrulation in Xenopus laevis. Development 130: 2129-2138.

OLIVARES G H, CARRASCO H, AROCA F, CARVALLO L, SEGOVIA F, LARRAIN J. (2009). Syndecan-1 regulates BMP signaling and dorso-ventral patterning of the ectoderm during early Xenopus development. Dev Biol 329: 338-349.

ORI A, WILKINSON M C, FERNIG D G. (2008). The heparanome and regulation of cell function: structures, functions, and challenges. Front. Biosci 13: 4309-4338.

PAINE-SAUNDERS S, VIVIANO B L, ZUPICICH J, SKARNES W C, SAUNDERS S. (2000). Glypican-3 controls cellular responses to Bmp4 in limb patterning and skeletal development. Dev Biol 225: 179-187.

PALMA V, CARRASCO H, REINCHISI G, OLIVARES G, FAUNES F, LARRAÍN J. (2011). Shh activity and localization is regulated by perlecan. Biol Res 44: 63-67.

SASAKI N, OKISHIO K, UI-TEI K, SAIGO K, KINOSHITA-TOYODA A, TOYODA H,
NISHIMURA T, SUDA Y, HAYASAKA M, HANAOKA, K, HITOSHI S, IKENAKA K NISHIHARA S. (2008). Heparan sulfate regulates self-renewal and pluripotency of embryonic stem cells. J Biol Chem 283: 3594-3606.

SCHNEIDER CA, RASBAND WS, ELICEIRI KW (2012). "NIH Image to ImageJ: 25 years of image analysis". Nature Methods 9: 671-675.

SEPICH, D S, USMANI M, PAWLLICKI S, SOLNICA-KREZEL L. (2011). Wnt/PCP signaling controls intracellular position of MTOCs during gastrulation convergence and extension movements. Development 138: 543-552.

SIVE H, GRAINGER R, HARLAND R. (2000). Early Development of Xenopus laevis: A Laboratory Manual. Cold Spring Harbor Laboratory Press.

SMITH, E M, MITSI M, NUGENT M A, SYMES K. (2009). PDGF-A interactions with fibronectin reveal a critical role for heparan sulfate in directed cell migration during Xenopus gastrulation. Proc Natl Acad Sci U S A 106: 21683-21688.

SMITH, J C. (2004). Role of T-box genes during gastrulation. In Gastrulation: From Cells to Embryo. (ed. C.D. Stern) Cold Spring Harbor Laboratory Press, Cold Spring Harbor, NY.

STICKENS D, ZAK B M, ROUGIER N, ESKO, J D, WERB Z. (2005). Mice deficient in ext2 lack heparan sulfate and develop extostoses. Development 132: 5055-5068.

TAKEI Y, OZAWA Y, SATO M, WATANABE A, TABATA T. (2004). Three Drosophila EXT genes shape morphogen gradients through synthesis of heparan sulfate proteoglycans. Development 131: 73-82.

TAO Q, YOKOTA C, PUCK H, KOFRON M, BIRSOY B, YAN D, ASASHIMAM, WYLIE C C, LIN X, HEASMAN, J. (2005). Maternal Wnt11 activates the canonical Wnt signaling pathway required for axis formation in Xenopusembryos. Cell120:857-871

VONICA, A, GUMBINER, B M. (2002). Zygotic Wnt activity is required for Brachyury expression in the early Xenopus laevis embryo. Dev Biol 250: 112-127.

YAMADA S, ONISHI M, FUJINAWA R, TADOKORO Y, OKABAYASHI K, ASASHIMA M, SUGAHARA K. (2009). Structural and functional changes of sulfated glycosaminoglycans in Xenopus laevis during embryogenesis. Glycobiology 19: 488-498

YAN D, LIN X. (2009). Shaping morphogen gradients by proteoglycans. Cold Spring Harb Perspect Biol 1: a002493.

YANAI I, PESHKIN L, JORGENSEN P, KIRSCHNER M W. (2011). Mapping gene expression in two Xenopus species: evolutionary constraints and developmental flexibility. Dev Cell 20: 483-496. 


\section{Further Related Reading, published previously in the Int. J. Dev. Biol.}

Xnr3 affects brain patterning via cell migration in the neural-epidermal tissue boundary during early Xenopus embryogenesis Mariko Morita, Satoshi Yamashita, Shinya Matsukawa, Yoshikazu Haramoto, Shuji Takahashi, Makoto Asashima and Tatsuo Michiue Int. J. Dev. Biol. (2013) 52: 161-161

http://dx.doi.org/10.1387/ijdb.130161tm

Direct control of Hoxd1 and Irx3 expression by Wnt/beta-catenin signaling during anteroposterior patterning of the neural axis in Xenopus

Sylvie Janssens, Tinneke Denayer, Tom Deroo, Frans Van Roy and Kris Vleminckx

Int. J. Dev. Biol. (2010) 54: 1435-1442

http://dx.doi.org/10.1387/ijdb.092985sj

Zygotic VegT is required for Xenopus paraxial mesoderm formation and is regulated by Nodal signaling and Eomesodermin

Masakazu Fukuda, Shuji Takahashi, Yoshikazu Haramoto, Yasuko Onuma, Yeon-Jin Kim, Chang-Yeol Yeo, Shoichi Ishiura and Makoto Asashima

Int. J. Dev. Biol. (2010) 54: 81-92

http://dx.doi.org/10.1387/ijdb.082837mf

Dicer inactivation causes heterochronic retinogenesis in Xenopus laevis

Sarah Decembrini, Massimiliano Andreazzoli, Giuseppina Barsacchi and Federico Cremisi Int. J. Dev. Biol. (2008) 52: 1099-1103

http://dx.doi.org/10.1387/ijdb.082646sd

Systematic screening for genes specifically expressed in the anterior neuroectoderm during early Xenopus development

Noriyuki Takahashi, Naoko Tochimoto, Shin-Ya Ohmori, Hiroshi Mamada, Mari Itoh, Masako Inamori, Jun Shinga, Shin-Ichi Osada and Masanori Taira

Int. J. Dev. Biol. (2005) 49: 939-951

http://dx.doi.org/10.1387/ijdb.052083nt

5 yr ISI Impact Factor $(2011)=2.959$
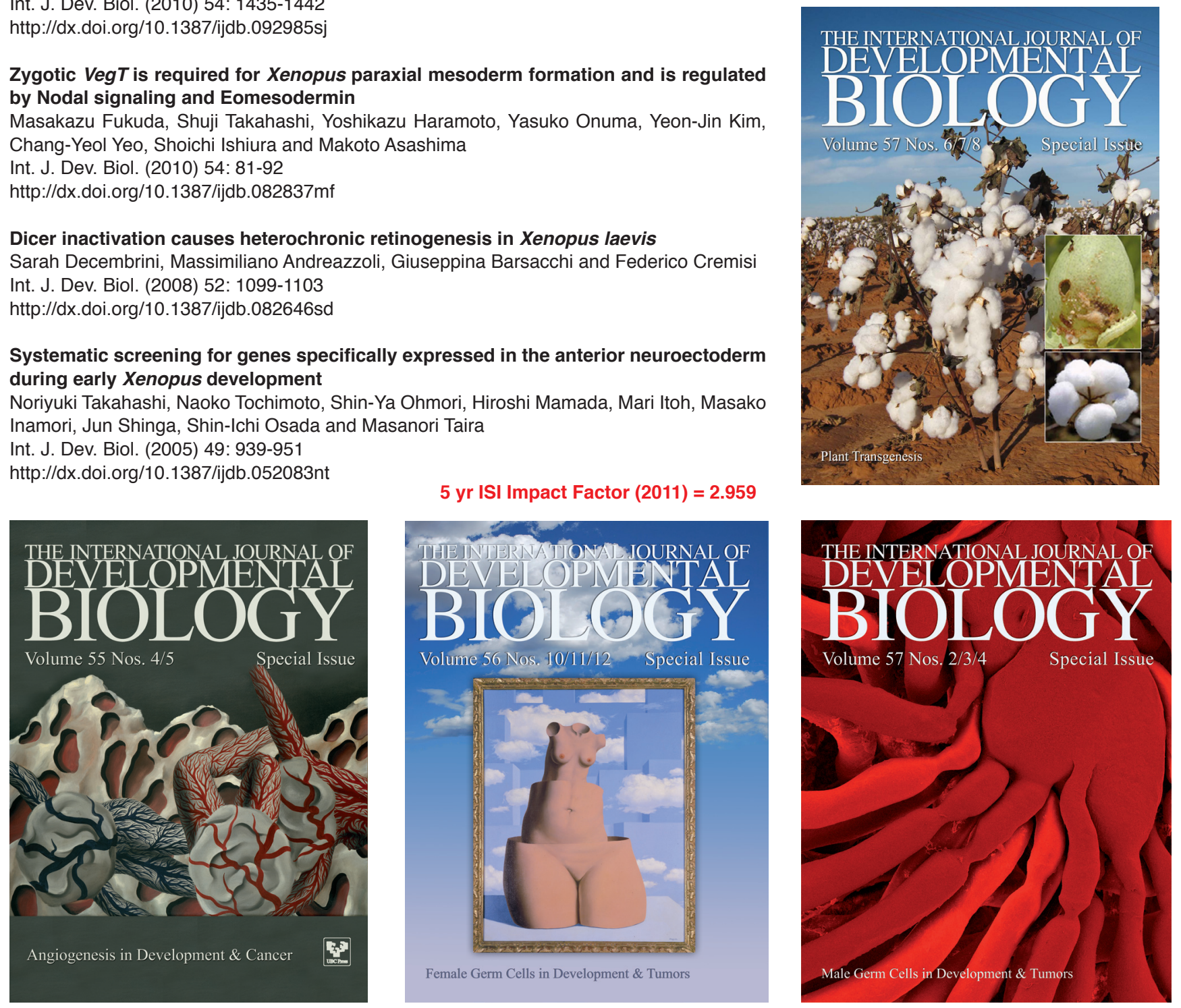